\title{
The antidepressant-like effect of almond oil: An additive effect with lavender oil.
}

\author{
Abdulrahman Al-Sayari' ${ }^{1}$, Mohammed Ghazwani², Yahya Alhamhoom ${ }^{2}$, Dalia Almaghaslah ${ }^{3}$, Justin V. \\ Louis $^{4}$, Narasimman Gurusamy ${ }^{*}$ \\ ${ }^{1}$ Department of Pharmacognosy, College of Pharmacy, King Khalid University, Abha-61441, Kingdom of Saudi Arabia \\ ${ }^{2}$ Department of Pharmaceutics, College of Pharmacy, King Khalid University, Abha-61441, Kingdom of Saudi Arabia \\ ${ }^{3}$ Department of Clinical Pharmacy, College of Pharmacy, King Khalid University, Abha-61441, Kingdom of Saudi \\ Arabia \\ ${ }^{4}$ Department of Pharmacology, College of Pharmacy, King Khalid University, Abha-61441, Kingdom of Saudi Arabia
}

\begin{abstract}
Background: Depression is a mood disorder that causes a persistent feeling of sadness and loss of interest in the usual activities. According to WHO, depression has been estimated to affect up to $21 \%$ of the world's population, while two recent cross-sectional studies conducted in the Kingdom of Saudi Arabia show that about $50 \%$ of the population is suffering from mild to severe depression.

Objective: Since the adverse effects associated with the current anti-depression medications are severe, the current study aimed to test the anti-depressant effect of natural oils, including almond oil, along with the inhalation of lavender oil, for alleviating depression.

Methods: Male Wister rats weighing approximately $250-300 \mathrm{~g}$ were used for the study. Almond oil was given to rats through oral or intraperitoneal injection $(3.2 \mathrm{~g} / \mathrm{kg})$. Inhalation of lavender oil (diluted with water; 1: 20) was given to rats for a period of 30 or $60 \mathrm{~min}$. The anti-depressant effect of the natural oils was evaluated using two different methods, either the forced swim test or the passive avoidance test.

Results: The anxiety and depression associated with forcing the rats to swim is reduced by the treatment with almond oil alone and in combination with the inhalation of lavender oil. The treatments resulted in the reduction of immobility events and an improvement in climbing behavior. Furthermore, the treatment of rats with almond oil alone and in combination with lavender oil inhalation increased the latency period to avoiding the shock in the passive avoidance test apparatus. The combination therapy resulted in a significant additive effect in the passive avoidance test.

Conclusion: Our results indicate that the treatment with almond oil or the combination of almond oil with the inhalation of lavender oil show anti-depressant-like effects in the rats studied, using the forced swim test and the passive avoidance test as evaluation tools.
\end{abstract}

Keywords: Depression, Antidepressant drugs, Almond oil, Lavender oil, Aroma therapy, Forced swim test, Passive avoidance test.

Accepted on March 27, 2018

\section{Introduction}

Depression is a common mental disorder and the leading cause of disability worldwide. It presents as depressed mood, loss of interest or pleasure, decreased energy, feelings of guilt or low self-worth, disturbed sleep or appetite, and poor concentration. Moreover, depression often comes with symptoms of anxiety. In general, women are more affected by depression than men. Furthermore, depression can lead to suicide. According to WHO, depression has been estimated to affect up to $21 \%$ of the world's population and it is expected to be the second most common disease in 2020 [1]. Two recent cross-sectional studies conducted in the Kingdom of Saudi Arabia show that about $50 \%$ of the population is suffering from mild to severe depression $[2,3]$.

Depression is associated with low levels of serotonin, in relation to norepinephrine and dopamine, in the brain. Currently, selective serotonin reuptake inhibitors, serotoninnoradrenaline reuptake inhibitors, and Tricyclic antidepressants are used for the therapeutic management of depression. However, the side effects associated with anti-depressant medications are numerous, including increased appetite, weight gain, sexual side effects, fatigue, drowsiness, insomnia, agitation, restlessness, and anxiety. [4,5]. 
In the treatment of depression, herbal therapies may be an effective alternative in terms of safety and tolerability, as well as improvement of patient compliance [4]. Many studies have addressed the effects of plant-derived essential oils on the central nervous system to relieve depression, anxiety, and stress [6,7]. Almond oil (Oleum amygdalae) has long been used in complementary medicine circles for its numerous health benefits, including anti-inflammatory, immunityboosting and anti-hepatotoxicity effects [8]. Moreover, cardiovascular benefits have also been identified with almond oil, such as elevating the levels of high-density lipoproteins while reducing low-density lipoproteins [9]. Historically, almond oil was used in Ancient Chinese, Ayurvedic and Greco-Persian Schools of Medicine to treat dry skin conditions such as psoriasis and eczema. Consumption of almond is shown to reduce the risk of colon cancer in a rat model of colon carcinogenesis [10]. Traditionally, almonds and almond oil have been used in several Asian countries as a memory booster. Hence, we hypothesized that almond oil may have anti-depressant-like characteristics. We tested the antidepressant effect of almond oil on rats using either a forced swim test or a passive avoidance test.

In addition to almond oil, lavender oil was also tested. Lavender has a high concentration of volatile oils, which impart its distinctive and pleasing fragrance. The relaxing experience of the lavender fragrance led to its therapeutic use in aromatherapy to relieve mild anxiety. Lavender has also been used internally for imbalances such as anxiety, insomnia, and gastrointestinal distress, including nervous stomach [11]. Lavender oil inhalation is shown to improve scopolamineinduced spatial memory impairment in laboratory rats [12]. Hence, we also hypothesized that the beneficial effects of almond oil can be synergized by aromatherapy with lavender oil.

\section{Materials and Methods}

\section{Materials}

The almond oil was manufactured by Divya Pharmacy, Haridwar, Uttarkhand, India, and the lavender oil (made in the UK) was purchased from the local supermarket in Abha, Saudi Arabia.

\footnotetext{
Animals

Male Wistar rats aged 2-3 months (body weight from $250 \mathrm{~g}$ to $300 \mathrm{~g}$ ) were maintained at $22-25^{\circ} \mathrm{C}$ at a relative humidity of 60 $\pm 20 \%$, under $12 \mathrm{~h}: 12 \mathrm{~h}$ light/dark cycles, with free access to food and water. This study was approved by the Animal Ethical Committee of King Khalid University, Abha and Kingdom of Saudi Arabia. All the animal experimental protocols were carried out in compliance with the Care and Use of Laboratory Animals.
}

\section{Almond oil treatment in rats}

The test animals were treated with either a low dose $(1.6 \mathrm{~g} / \mathrm{kg})$ or a high dose $(3.2 \mathrm{~g} / \mathrm{kg})$ of almond oil via intraperitoneal (i.p.) injection, while the control animals were injected with volumematched normal saline via i.p. injection. Oral administration of almond oil was given at the dose of $3.2 \mathrm{~g} / \mathrm{kg}$. $30 \mathrm{~min}$ after drug administration, the animals underwent either a forced swim test or a passive avoidance test.

\section{Lavender oil aromatherapy in rats}

Lavender oil obtained from the local supermarket was diluted with double distilled water (1:20), and heated in an oil burner using a candle. The animals were placed in a large cage with the oil burner placed atop to ensure that the animals could not touch the burner. The animal cage and the burner were covered with another plastic container. The top plastic container was briefly open every 10 min to check that the burner was on and to allow air into the cage. The lavender oil aromatherapy was given either for a period of $30 \mathrm{~min}$ (low dose) or $60 \mathrm{~min}$ (high dose). The control animals were treated with steam for the respective periods of time. After the treatment periods, the animals underwent either a forced swim test or a passive avoidance test.

\section{The combination therapy of almond oil and lavender oil}

For the combination therapy, the animals were provided with the inhalation of either lavender oil or steam for a period of 30 minutes. Then, the animals were given the intraperitoneal injection of either almond oil $(3.2 \mathrm{~g} / \mathrm{kg})$ or volume-matched normal saline. Further, the animals were allowed to continue their respective inhalations for a period of another $30 \mathrm{~min}$. At the end of these treatments, the animals undertook either a forced swim test or a passive avoidance test.

\section{Forced swim test}

The forced swim sessions were conducted by placing the rats individually in large glass cylinders $(45 \times 20 \mathrm{~cm})$ containing $24-25^{\circ} \mathrm{C}$ water approximately $30 \mathrm{~cm}$ deep. The water was sufficiently high so that the rats could not escape or touch the bottom of the beaker. Each rat was placed in the cylinder for 5 min, followed by intraperitoneal drug administration. Thirty minutes later, a $5 \mathrm{~min}$ forced swim test was conducted, and during the test, the animals were videotaped. The videotapes were analyzed for time spent on climbing, swimming, and immobility. During the entire duration of the task, an experimenter was present and watching the rats. If there was any indication that an animal was in danger of drowning, it was immediately removed from the beaker and excluded from the study. At the end of the swim session, the rats were towel dried and placed in a clean cage. The water in the test beaker was changed for each subject. 


\section{Passive avoidance test}

The passive avoidance test apparatus consisted of a twocompartment box $(24 \times 16 \times 20 \mathrm{~cm}$ for each compartment $)$. The front chamber was illuminated with a $40-\mathrm{W}$ bulb (white box) and connected to the rear dark chamber, which is equipped with a grid floor. The two chambers are separated by a door. On day 1 , the test rats are placed individually into the illuminated compartment and allowed to explore the boxes. The latency to enter the dark chamber is recorded. On day 2, the animals are again placed in the light chamber, and upon entry into the dark chamber, receive a mild foot shock (1 mA) of $3 \mathrm{~s}$ duration. On day 3 , the rats are treated with either almond oil injected intraperitoneally (at $3.2 \mathrm{~g} / \mathrm{kg}$ ), and/or with lavender oil inhalation (60-min of duration, as established), followed by placement in the light chamber. The latency to enter the dark chamber is recorded. If the animal has not entered the dark chamber after $180 \mathrm{~s}$, it is returned to its cage and the latency is recorded as greater than $180 \mathrm{~s}$.

\section{Statistics}

All values are expressed as the mean \pm standard error of mean. Student's t-test was performed to compare the differences between the control and treatment groups. The results were considered significant if $\mathrm{P}<0.05$.

\section{Results}

\section{The dose-dependent anti-depressant effect of almond oil in rat FST}

Swimming behaviors such as climbing, swimming and immobility were analyzed during the forced swim test in rats. As noted in Figure 1A, the climbing behavior in the high-dose almond oil group was significantly $(\mathrm{P}<0.05)$ increased (up to $102 \mathrm{~s}$ ) compared to the control group (44 s), whereas the swimming behavior in the high-dose almond oil group was slightly increased (up to $128 \mathrm{~s}$ ) compared to the control group (120 s). Importantly, the immobility behavior is significantly reduced $(\mathrm{P}<0.01)$ to $55 \mathrm{~s}$ in the high-dose almond oil group compared to its respective control group (120 s) (Figure 1A).

On the other hand, as seen in Figure 1B, the climbing behavior in the low-dose almond oil group was slightly increased (up to $70 \mathrm{~s})$ compared to the control group (62 s), whereas the swimming behavior in the high-dose almond oil group was slightly increased (up to $110 \mathrm{~s}$ ) compared to the control group (104 s). Furthermore, the immobility behavior was moderately reduced to $112 \mathrm{~s}$ in the low-dose almond oil group compared to its respective control group (142 s) (Figure 1B).

\section{Comparative effect of intraperitoneal and oral dose of almond oil in rat FST}

As shown in Figure 1C, the anti-depressant effects of intraperitoneal and oral doses of almond oil were compared in rat FST. We found that the climbing behavior of rats was significantly improved by the oral dose of almond oil compared to the control rats, whereas the swimming and immobility behaviors were not significantly altered by the oral dose of almond oil (Figure 1C). It is to be noted that the intraperitoneal dose of almond oil significantly reduced the immobility behaviors, whereas the same dose of oral almond oil was not effective in reducing the immobility behaviors in rat FST, although the oral dose was effective in improving the climbing behaviors of rats (Figure 1C).

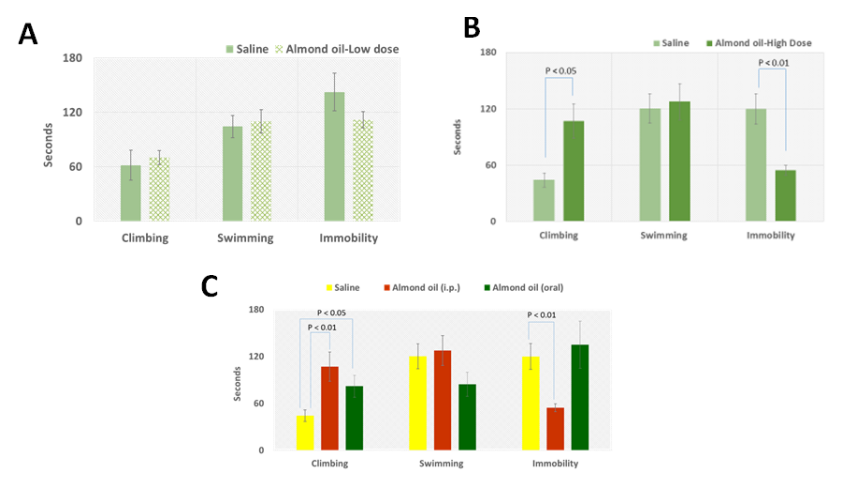

Figure 1. The effect of almond oil inhalation in rat forced swim test. A) The effect of low-dose almond oil $(1.6 \mathrm{~g} / \mathrm{kg}$; injection via i.p.; $n=6) ; B)$ The effect of high-dose almond oil $(3.2 \mathrm{~g} / \mathrm{kg}$; injection via i.p.; $n=6$ ), compared with its respective controls ( $n=6$ each, volumematched i.p. injection of normal saline) in the swimming behaviors (climbing, swimming and immobility) during the forced swim test in rats; C) Comparative effect of intraperitoneal $(3.2 \mathrm{~g} / \mathrm{kg} ; \mathrm{n=6})$ and oral dose $(3.2 \mathrm{~g} / \mathrm{kg} ; n=6)$ of almond oil in rat forced swim test.

\section{The dose-dependent anti-depressant effect of inhalation of lavender oil in rat FST}

Inhalation of lavender oil was given to the rats for either 30 min (low-dose) or $60 \mathrm{~min}$ (high-dose) as mentioned. Subsequently, the rats were tested for their swimming behaviors, such as climbing, swimming and immobility, in the forced swim test. As shown in Figure 2A, the climbing behavior in the low-dose lavender oil group was slightly higher than the control group, but it was significantly $(\mathrm{P}<0.05)$ higher in the high-dose lavender oil group. The swimming behavior in the high-dose lavender oil group was slightly higher than the low-dose lavender and control groups, but this difference was not statistically significant (Figure 2A). More importantly, the immobility behavior was significantly $(\mathrm{P}<0.05)$ reduced in the high-dose lavender oil group compared to its respective control group, while this behavior was moderately (not significantly) reduced in the low-dose lavender oil group compared to its respective control group (Figure 2A).

\section{The effect of combination therapy (almond oil and inhalation of lavender oil) in rat FST}

For the combination therapy, we used $3.2 \mathrm{~g} / \mathrm{kg}$ of i.p. almond oil and $60 \mathrm{~min}$ lavender oil inhalation for rats, as described in the Methods section. Figure 2B illustrates that the combination therapy resulted in an increased amount of time spent in climbing compared with either therapy alone. 
As expected, the combination therapy resulted in a statistically significant $(\mathrm{P}<0.001)$ improvement in climbing compared with the control group, but the However, the additive effect, or synergistic effect, of the combination therapy was not seen for (Figure 2B).

However, the swimming behavior was similar among the groups (Figure 2B). The immobility behavior was significantly $(\mathrm{P}<0.001)$ reduced in the combination therapy group compared to its respective control group, but the combination therapy did not produce any additive effect in reducing the immobility behavior as compared with the individual treatments of almond oil or lavender oil (Figure 2B).
A

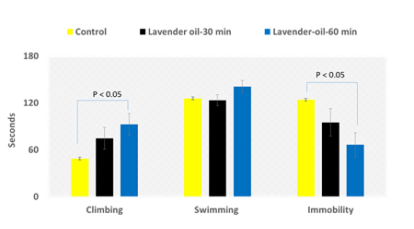

B

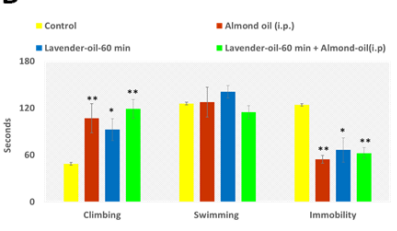

Figure 2. The effect of lavender oil inhalation in rat forced swim test. A) Inhalation of lavender oil was given to rats for the period of either $30 \mathrm{~min}(n=6)$ or $60 \mathrm{~min}(n=7)$, and the results are compared with their control animals $(n=4)$ treated with steam for the respective period, as mentioned in the methods section; B) The groups of animals were treated with either almond oil $(3.2 \mathrm{~g} / \mathrm{kg}$; injection via i.p.; $n=6$ ) or volume-matched normal saline (control) given via i.p. or inhalation of lavender oil for a period of $60 \mathrm{~min}(n=6)$ or the combination of both almond oil and lavender oil inhalation $(n=8)$ as mentioned in the methods section. ${ }^{*} P<0.05 ;{ }^{* *} P<0.001$ vs. their respective control group.

\section{The antidepressant effect of almond oil in combination with lavender oil inhalation in the passive avoidance test}

Almond oil at a dosage of $3.2 \mathrm{~g} / \mathrm{kg}$ was injected i.p. $30 \mathrm{~min}$ before testing the animal in the passive avoidance test apparatus.

As shown in Figure 3A, our results clearly indicate that the average latency time significantly $(\mathrm{P}<0.05)$ increased in the rats treated with almond oil (32.4 s) compared to the control animals $(7.8 \mathrm{~s})$ treated with normal saline.

The inhalation of lavender oil was given for $60 \mathrm{~min}$ before undertaking the passive avoidance test. Figure $3 \mathrm{~B}$ notes that the average latency time significantly $(\mathrm{P}<0.05)$ increased in the rats treated with the inhalation of lavender oil $(95.1 \mathrm{~s})$ compared to the control animals (33.8 s) treated with steam inhalation.

Furthermore, the treatment with the combination of almond oil and inhalation of lavender oil significantly $(\mathrm{P}<0.01)$ increased the average latency period (134.1 s) when compared with the control rats (57.3) treated with the combination of normal saline and steam.

Based on the average latency period (Figures 3A-3C), the combination of almond oil and lavender oil treatment resulted in an additive effect in the passive avoidance test (134.1 s) when compared to either almond oil (32.4 s) or the inhalation of lavender oil (95.1 s) alone.

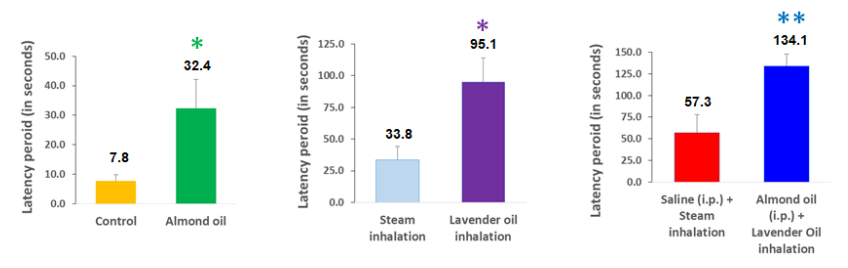

Figure 3. The effect of almond oil or the inhalation of lavender oil or the combination of both in passive avoidance test. The rats underwent a passive avoidance test, as mentioned in methods. A) On day 3, the animals were given either almond oil $(3.2 \mathrm{~g} / \mathrm{kg}$; injection via i.p.; $n=11)$ or volume-matched normal saline (control; $n=10)$ given via i.p. for a period of $30 \mathrm{~min}$. At the end of the treatment period, the treated animals underwent a passive avoidance test, and the latency period to enter the dark chamber was measured; B) On day 3, the animals were given either inhalation of lavender oil for $60 \mathrm{~min}$ $(n=11)$ or inhalation of steam for $60 \mathrm{~min}$ (control; $n=7)$. At the end of the treatment period, the treated animals underwent a passive avoidance test, and the latency period to enter the dark chamber was measured; C) On day 3, the animals were given the combination of almond oil and inhalation of lavender oil $(n=15)$ or the combination of normal saline and inhalation of steam $(n=15)$, as mentioned in the methods section. At the end of the treatment period, the treated animals underwent a passive avoidance test, and the latency period to enter the dark chamber was measured. ${ }^{*} P<0.05$; ${ }^{* *} P<0.001$ vs. their respective control group.

\section{Discussion}

The forced swim test, or the behavioural despair test, is the most commonly used test to measure the effects of antidepressant treatment [13]. The characteristic behavior of the test, termed immobility, develops when a rodent is placed in a tank of water for an extended period of time and "makes only those movements necessary to keep its head above water." The development of immobility is usually facilitated by a pretest of $5 \mathrm{~min}$ given $30 \mathrm{~min}$ before a 5 -min test. When antidepressants are administered between the pre-test and the test, the antidepressant drugs are expected to decrease immobility. The current study results clearly demonstrate that the anxiety or depression associated with the forced swimming is reduced, as evidenced by the reduction of immobility events in the rats treated with high-dose almond oil. Moreover, the motor skills, such as swimming and climbing, are improved in rats treated with high-dose almond oil. Though some improvements were seen in low-dose almond-oil, the differences were not statistically significant.

Almond oil contains sizeable proportions of essential fatty acids, which need to be derived from the diet. They are deemed essential, since they are not synthesized by the body. Almond oil is rich in beta-zoosterol, squalene and alpha-tocopherol, all of which are important constituents of healthy-looking skin. Interestingly, the almond's rich vitamin B complex and zinc content also support its skin-beautifying properties, as these components are key to developing and maintaining healthylooking skin [14]. Although the component that is responsible for the anti-depressant characteristics is not known, a recent 
study indicates that omega-3 fatty acids have anti-depressantlike effects [15]. This study reported that the fatty acids present in menhaden fish oil, which is rich in omega-3, exerted a beneficial effect on postpartum depression and decreased the biomarkers related to depression such as corticosterone. It also reduced the pro-inflammatory cytokines [15].

Learned helplessness, a technical term in both animal and human psychology, refers to a condition in which a human or animal has learned to behave helplessly, even when an opportunity to help itself to avoid an unpleasant or harmful circumstance is presented. The concept of "helplessness" was first used to describe the failure of some laboratory animals to escape or avoid shock when given the opportunity, after previous exposure to inescapable shock $[16,17]$. The learned helplessness theory is seen in clinical depression and related mental illnesses when a person perceives the absence of control over the outcome of a situation. This theory has been established as a model for depression since behavioural correlates of helplessness are seen as a frequent feature in depressed patients; thus, its pharmacological specificity is included for antidepressant drug treatment. Avoidance tasks are aversively motivated escape paradigms. Passive avoidance requires the subject to remember the properties of a location in which a foot shock was received the previous day, and to refrain from entering that location. Avoidance tasks are sensitive to hippocampal lesions and pharmacological treatments with antidepressant and cholinergic drugs [18,19].

In the current study, our results from a passive avoidance test clearly indicate that the average latency time was significantly increased in the rats treated with either almond oil or inhalation of lavender oil. Furthermore, an additive effect was found with the combination therapy using the two chosen oils.

In vitro and in vivo studies have demonstrated multiple possible mechanisms of action for lavender oil, as well as its individual constituents, which may partly account for its relaxing effects when taken orally. Lavender oil has indicated potentiated expression of GABA-A receptors in cell culture and has shown spasmolytic activity on guinea pig ileum [20,21]. Linalool, a main active ingredient in lavender oil, has been shown to inhibit glutamate binding in animal brains and has also inhibited acetylcholine release and influenced ionic conductance in neurons [22,23]. Additionally, linalyl acetate has been reported to exert a relaxing effect [24]. Lavender oil has reduced dose-dependent spontaneous motility and the caffeine-induced hyperactivity of mice [25].

The hippocampus region is responsible for the memory of new information and recent events. Hippocampal shrinkage is a commonly found neuroanatomical change in stress-related mood disorders, such as depression and post-traumatic stress disorders (PTSD). Adult neurogenesis is regulated or modulated by exogenous and endogenous factors. These factors up-regulate or down-regulate the cellular processes of adult neurogenesis. Based on animal studies, changes in hippocampal volume have been correlated to other factors, such as enriched environment, behavioural stress and depression, genetics and growth factors, disease, neuroendocrine early life experiences, physical exercise and neurochemical factors. These factors suggest that the changes observed in the hippocampal volume relate to an increase/ decrease in hippocampal neurogenesis [26,27].

In conclusion, our study results clearly indicate that treating rats with either almond oil or lavender oil inhalation, or a combination of both, reduced the immobility characteristics associated with depression in the forced swim test, and increased the latency period to avoid a shock in the passive avoidance test apparatus. This implies that a treatment with almond oil, inhalation of lavender oil, or both might enhance the function of the hippocampal region.

\section{References}

1. WHO. Depression. World Health Organization, Geneva 2006.

2. Waleed A-Q, Saeed U-R, Mazen SF, Imad AA. Adult depression screening in Saudi primary care: prevalence, instrument and cost. BMC Psychiatry 2014; 14: 190.

3. Abdulaziz UJ, Abdulaziz AA, Sara AA, Mohammed A, Norah AA, Abdullah BM, Mohammed AA. Prevalence of depression and its association with socio-demographic characteristics among the general population. Int J Modern Alternative Med Res 2014; 2: 8-15.

4. Richelson E. Pharmacology of antidepressants characteristic of the ideal drug. Mayo Clinic Proceedings 1994; 69: 1069-1081.

5. Taylor S, Stein MB. The future of selective serotonin reuptake inhibitors (SSRIs) in psychiatric treatment. Med Hypotheses 2006; 66: 14-21.

6. Almeida RN, Araújo DAM, Gonçalves JCR, Montenegro FC, Sousa DP, Leite JR, Mattei R, Benedito MAC, Carvalho JGB, Cruz JS, Maia JGS. Rosewood oil induces sedation and inhibits compound action potential in rodents. J Ethnopharmacol 2009; 124: 440-443.

7. Seol GH, Shim HS, Kim PJ, Moon HK, Li KH, Shim I, Suh $\mathrm{SH}$, Min SS. Antidepressant-like effect of Salvia sclarea is explained by modulation of dopamine activities in rats. J Ethnopharmacol 2010; 130: 187-190.

8. Ahmad Z. The uses and properties of almond oil. Complement Ther Clin Pract 2010; 16: 10-12.

9. Jenkins DJ, Kendall CW, Marchie A, Parker TL, Connelly PW, Qian W. Dose response of almonds on coronary heart disease risk factors: blood lipids, oxidized low-density lipoproteins, lipoprotein a, homocysteine, and pulmonary nitric oxide: a randomized, controlled, crossover trial. Circulation 2002; 106: 1327-1332.

10. Davis PA, Iwahashi CK. Whole almonds and almond fractions reduce aberrant crypt foci in a rat model of colon carcinogenesis. Cancer Lett 2001; 165: 27-33.

11. Blumenthal M. Lavender flower In: The complete German commission e monographs. American Botanical Council, Austin, Texas, USA 1998; 159-160.

12. Hritcu L, Cioanca O, Hancianu M. Effects of lavender oil inhalation on improving scopolamine-induced spatial 
memory impairment in laboratory rats. Phytomedicine 2012; 19: 529-534.

13. Castagné V, Moser P, Roux S, Porsolt RD. Rodent models of depression: Forced swim and tail suspension behavioral despair tests in rats and mice. Curr Protocols Pharmacol $2010 ; 49$.

14. Kuriyama H, Watanabe S, Nakaya T, Shigemori I, Kita M, Yoshida N. Immunological and psychological benefits of aromatherapy massage. Evid Based Complement Alternat Med 2005; 2: 179-184.

15. Arbabi L, Baharuldin MT, Moklas MA, Fakurazi S, Muhammad SI. Antidepressant-like effects of omega-3 fatty acids in postpartum model of depression in rats. Behav Brain Res 2014; 271: 65-71.

16. Ovemier JB, Seligman M. Effects of inescapable shock upon subsequent escape and avoidance learning. J Comp Physiol Psychol 1967; 63: 28-33.

17. Ying X, Philip AB, Jianchun P, William OO, James MO'D. Animal models of depression and neuroplasticity: assessing drug action in relation to behavior and neurogenesis. In methods in molecular biology: psychiatric disordersmethods and protocols. Humana Press, Gainesville, FL, USA 2012.

18. Redmond AM, Kelly JP, Leonard BE. The determination of the optimal dose of milnacipran in the olfactory bulbectomized rat model of depression. Pharmacol Biochem Behav 1999; 62: 619-623.

19. Xu Y, Ku BS, Yao HY, Lin YH, Ma X, Zhang YH, Li XJ. Antidepressant effects of curcumin in the forced swim test and olfactory bulbectomy models of depression in rats. Pharmacol Biochem Behav 2005; 82: 200-206.

20. Aoshima H, Hamamoto $K$. Potentiation of GABAA receptors expressed in Xenopus oocytes by perfume and phytoncid. Biosc Biotechnol Biochem 1999; 63: 743-748.
21. Lis-Balchin M, Hart S. Studies on the mode of action of the essential oil of lavender. Phytother Res 1999; 13: 540-542.

22. Elizabetsky E, Al M. Effects of linalool on glutamatergic system in the rat cerebral cortex. Neurochem Res 1995; 20: 461-465.

23. Re L, Barocci S, Sonnino S. Linalool modifies the nicotinic receptor-ion channel kinetics at the mouse neuromuscular junction. Pharmacol Res 2000; 42: 177-182.

24. Tisserand R, Balacs T. Essential oil safety. A guide for health care professionals. Harcourt, Glasgow, UK 1999.

25. Buchbauer G, Jirovetz L, Jager W, Dietrich H, Plank C. Aromatherapy: evidence for sedative effects of the essential oil of lavender after inhalation. Z Naturforsch C 1991; 46: 1067-1072.

26. Bergami M, Masserdotti G, Temprana Silvio G, Motori E, Eriksson Therese M, Göbel J, Yang Sung M, Conzelmann KK, Schinder Alejandro F, Götz M, Berninger B. A critical period for experience-dependent remodeling of adult-born neuron connectivity. Neuron 2015; 85: 710-717.

27. Loi M, Koricka S, Lucassen P, Joels M. Age and sexdependent effects of early life stress on hippocampal neurogenesis. Front Endocrinol 2014; 5: 13.

\section{*Correspondence to}

Narasimman Gurusamy

Department of Pharmacology

College of Pharmacy

King Khalid University

Abha-61441

Kingdom of Saudi Arabia 